\title{
A New Mixed Signal Platform to Study the Accuracy/Complexity Trade-Off of DPD Algorithms
}

\author{
Hanan Thabet \\ Department of Signal Processing \& \\ Electronic Systems \\ Centrale Supélec \\ Gif Sur Yvette, FRANCE
}

\author{
Morgan Roger \\ Department of Signal Processing \& \\ Electronic Systems \\ Centrale Supélec \\ Gif Sur Yvette, France
}

\author{
Caroline Lelandais-Perrault \\ Department of Signal Processing \& \\ Electronic Systems \\ Centrale Supélec \\ Gif Sur Yvette, FRANCE
}

\begin{abstract}
The increase in bandwidth of Power Amplifier (PA) input signals has led to the development of more complex behavioral PA models. Most recent models such as the Generalized Memory Polynomial (1) or the Polyharmonic distortion modeling (2) can be used to design very performant but complex and thus very consuming Digital Predistortion algorithms (DPDs). On the other hand, with earlier simpler models, the precision of the DPD may not be enough. The model order is also the major factor influencing the requirements in terms of bandwidth and dynamic range of the digitized signal in the feedback loop of a typical Power amplification system architecture: the higher the order, the more information is needed for identification.

This paper describes a new mixed signal simulation platform developed to study the complexity vs. accuracy trade-off from the DPD point of view. The platform estimates the accuracy of the DPD and the power consumption (including the consumption of the DPD itself) of the whole feedback loop, by comparing various PA models with various DPDs algorithms. Contrary to older works, measuring the accuracy on the open loop without DPD and estimating the complexity in theoretical number of operations, our goal is to be able to estimate with precision the performances and the power consumption of the whole amplification system (PA + DPD + DAC + feedback loop) for optimization of DPD algorithms.
\end{abstract}

Keywords-LTE; DPD algorithms; Simulation Platform; Accuracy/Complexity; Power Consumption; Co-Simulation

\section{INTRODUCTION}

The increase of mobile services based on 4G LTE services across global markets, provides subscribers with the type of responsive Internet browsing experience that previously was only possible on wired broadband connections. In fact, LTE has a rapid uptake since LTE subscriptions are expected to exceed 1.3 billion by the end of 2018 (3), while they does not exceed more than 200 commercial LTE networks in operation as of August 2013 (3). Furthermore, with the exponential growth in network data traffic, the mobile industry must offers products with higher capabilities and performances with lower cost than existing wireless systems, including LTE (4). This strategy will enable new services, new applications and thus must be based on a combination of network topology innovations and new terminal capabilities. To address these requirements, wireless transmission systems, operating at higher frequencies, requires more bandwidth and increases the demands on the linearity of power amplifiers (PAs). Moreover, with the growth of power consuming base stations' number, it is very important to develop power-efficient linear devices in radio base stations (5). Since for mobile devices the power is driven from limited battery supplies, the power consumption is the most critical parameter to optimize. As the RF power amplifiers (PAs) are the most power consuming building blocks in wireless transmitters, their design is highly constrained by trade-off between power efficiency and linearity. This is why, in recent years, the interest in PA modeling has increased. In this paper, the second session describes the state of the art and advancements of one of the most promising linearization techniques.

In Sections III, IV and V, we describe three platforms developed in order to study the complexity/efficiency trade-off for LTE base stations.

\section{PA LINEARIZATION TECHNIQUES}

\section{A. PA characteristics}

In fact, high efficiency operating PA induces unacceptable nonlinear distortions at the output of the PA. On the other hand, the price for linearity is a sharp drop in power efficiency (6). So, power efficiency and linearity present key parameters of amplification systems but they cannot be achieved simultaneously. This is why it is necessary to apply linearization techniques to a high-performance amplifier but not sufficient linear. Many techniques have been developed to achieve high efficiency linear operating modes of PAs, but the main drawback of such techniques comes from the fact that the generated waveforms are extremely vulnerable to imperfections in the transmission chain (shown in Figure 1). Some of these techniques were dedicated to improve efficiency behaviour and others to extend the linear behaviour into high efficiency regions of operation. The latter's are known as linearization techniques.

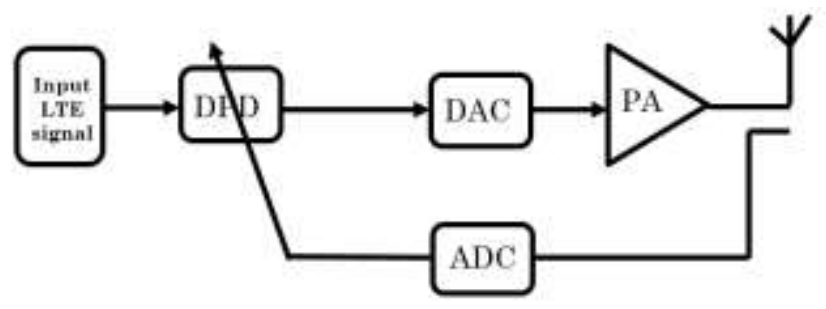

Fig. 1. Typical Power amplification system architecture 
An amplifier is linear if its gain is constant throughout the range of the input signal. If this gain is not linear, the output signal is distorted by clipping. The DC bias point is the most important factor in determining the relationship between PA nonlinearity and efficiency. For a real amplifier, the gain and phase-shift are functions of the input signal. The complex transfer function is dependent on the amplifier input power. As a result, the gain decreases and phase-shift changes as the level of the input signal drives the amplifier into its saturation region. The PA output amplitude and phase characteristics are known as AM-AM (Amplitude-to-Amplitude) and AM-PM (Amplitude-to-Phase) characteristics, respectively (7). AM/AM and AM/PM are typical way to characterize the nonlinearity of a PA. An example of AM/AM and AM/PM characteristics is shown in Figure 2. Unfortunately, to model a real PA there is an important parameter to characterize which is the Power Added Efficiency (PAE) (Figure 3). At the base station, linearity in the PA is more important than efficiency (8).

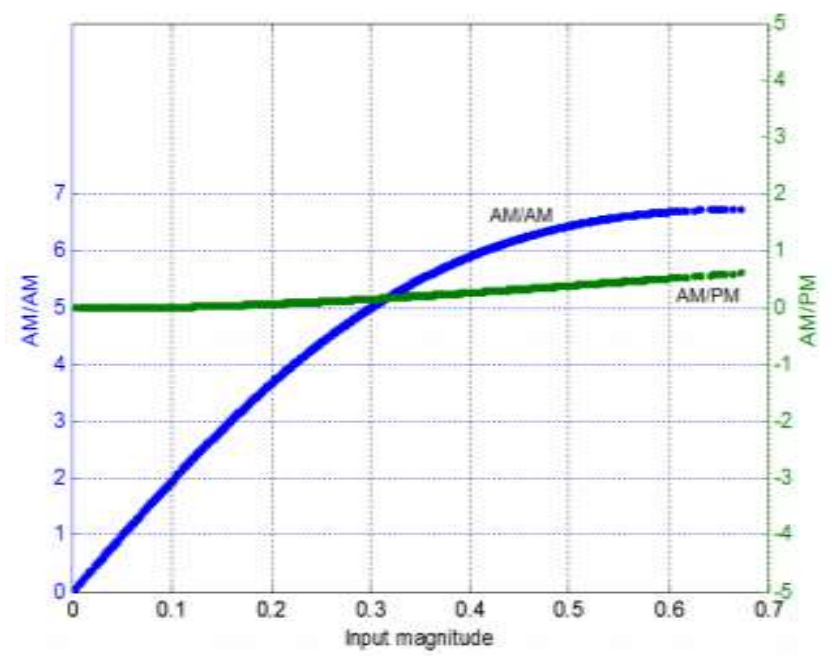

Fig. 2. AM/AM and AM/PM characteristics for a Typical PA

\section{B. Digital Predistortion Technique}

In fact, there are various linearization techniques which are classified according to their functionality, architecture, and application. Details of these schemes and their techniques are described in (8) and (10).

The digital predistortion technique (DPD) is considered as a key enabling technique for future radio transmitters. It is entirely implemented using digital processors and this offers re-configurability capabilities, one of the main features of future communication systems. This common technique provides an inexpensive solution in which a nonlinear circuit is inserted between the input signal and the PA. The nonlinear circuit generates IMD products inverse to that produced by the PA and thereby cancels the effect of the PA nonlinearity.

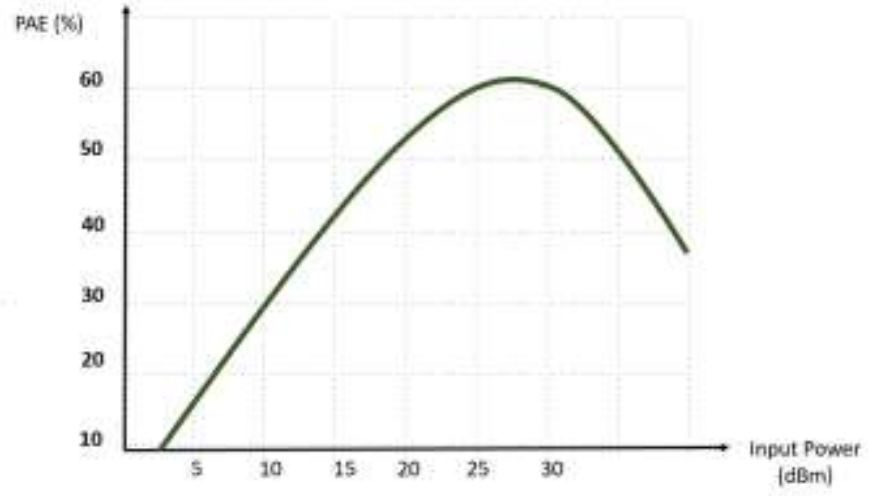

Fig. 3. PA Power Added Efficiency (PAE)

This can be viewed as the predistorter having characteristics inverse to the real PA AM-AM curve in Figure. 1. So DPD is a baseband signal processing technique that corrects impairments of RF PAs. These impairments cause outof-band emissions, spectral regrowth and in-band distortion of Wideband signals with a high peak-to-average ratio. LTE/4G transmitters, are particularly susceptible to these unwanted effects. The Error Vector Magnitude (EVM) and Peak-toAverage Power Ratio (PAPR) metrics are measured to evaluate the power efficiency and the linearity of an amplification system (figure .1). As shown in Figure 4, DPD improves the adjacent channel power ratio (ACPR) caused by impairments already discussed.

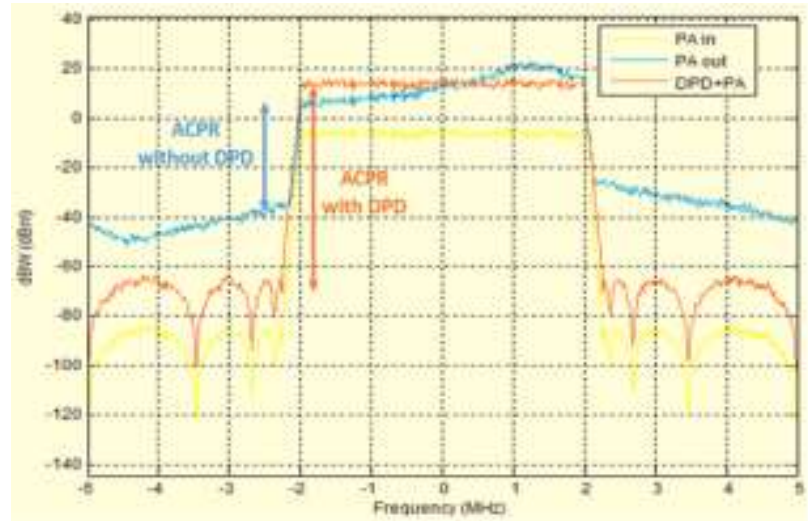

Fig. 4. Example of Adjacent channel power ratio (ACPR) improvement with DPD

\section{MATLAB PlatForm}

To simplify the implementation of the platform developed and to solve various problems that may be encountered, we have chosen to divide the implementation into three dependent stages or steps The first Matlab platform is developed in Matlab/Simulink to implement an adaptive DPD algorithm. This platform uses Simulink block circuits to model the chain 
presented in Figure 1. The modeling, itself, was divided into three parts and is based on some simplifications to accelerate simulations. In fact, the PA is an analog circuit using a real pass-band signal while we use a base-band complex signal for a discrete time system. Furthermore, we do not include quantization effects due to the ADC and DAC and up/down conversion effects. First, we model the PA using a polynomial memoryless Saleh amplifier described in (12) followed by an asymmetrical complex filter. The PA simulation then extracts the system inputs and outputs that will be used later to find the expression of the DPD algorithm. Equation (1) describes a memory polynomial (11) form for a non-linear PA:

$$
y_{M P}(n)=\sum_{k=0}^{K-1} \sum_{m=0}^{M-1} a_{k m} x(n-m) \mid x(n-m)^{k}
$$

Where $\mathrm{x}$ is the PA complex input, $\mathrm{y}$ is the PA complex output, akm are the PA polynomial complex coefficients, $\mathrm{M}$ is the PA memory depth, $\mathrm{K}$ is the degree of PA non-linearity and $\mathrm{n}$ is the time index. The next part consists on deriving DPD coefficients using matrix inverse algorithm by reversing the roles of $\mathrm{x}$ and $\mathrm{y}$ in equation (1) as the DPD is the inverse nonlinear function. So we can derive DPD coefficients using this equation (2):

$$
\left.x_{M P}(n)=\sum_{k=0}^{K-1} \sum_{m=0}^{M-1} d_{k w+} u(n-m)\right\rfloor(n-m)^{k}
$$

Where $d_{k m}$ are DPD coefficients used for implementing equation (2) in Matlab for a static DPD. The last part consists of extending the static DPD design to adaptive one based on Least Mean Square (LMS) algorithm in order to evaluate a functional validation of the amplification system and provide an estimation of the ACPR and the EVM. The LMS algorithm used an indirect learning architecture to implement an adaptive DPD with no matrix inverse. Figure 4. Shows an example of curve's Output spectrum using the platform Matlab/Simulink simulation for a system with $\mathrm{K}=\mathrm{M}=5$.

\section{MATLAB/CADENCE PLATFORM}

This second Matlab/Cadence platform was developed to estimate with more accuracy ACPR, EVM and the analog part power consumption. So, the design of the analog part of the amplification system was done on Cadence using Verilog A language and modifying models of the PA, mixers, VCO, ADC and DAC available in Cadence library. This platform shown in Figure 5. Is based on a Matlab/Cadence cosimulation using couplers and saving the DPD algorithm already developed and presented in section III for the Matlab platform. Figure 6. Shows an example of simulation results of co-simulation using the AMS designer as Cadence simulator. In fact, the high level system concept is often specified in the early stages of design work. This process is well supported by both concept engineering and system-level simulation tools such as MATLAB/Simulink. Co-simulation using AMS designer and MATLAB/Simulink combines the best of systemlevel simulation with lower-level analog and RF simulation. Simulink provides large libraries of DSP algorithms for generating complicated signals and post processing while AMS supports transient and envelope analysis of RF and communication circuits, such as mixers and oscillators both at the transistor and behavioral levels. Therefore, to run the cosimulation, Cadence recommends using specific software versions and some libraries and blocks must be installed for the two tools such as Couplers (13).

\section{CAdence Ultimate PlatForm}

The PA, in the previous platform, modeled in Verilog A does not include the AM/PM distortion, therefore, it was necessary to add a filter after the PA. Furthermore, the power consumption of the analog part does not be accurate estimated. So, we implement the third Cadence Platform with the RF analog part in Transistor Level and Digital part in VHDL to obtain accurate estimation of power consumption of digital part (DPD algorithm). But, by using the AMS designer simulator, Harmonic Balance simulation cannot be established to visualize spectrums and deduce ACPR, EVM and DPD parameters. As a solution, we develop Post processing in Matlab using Cadence simulation results. In fact, we need also to improve PA model by including memory effects and the recursive adaptive DPD algorithm in VHDL. So to design the $\mathrm{RF}$ analog part at transistor level, we use the $0.35 \mu \mathrm{m}$ AMS CMOS standard technology.

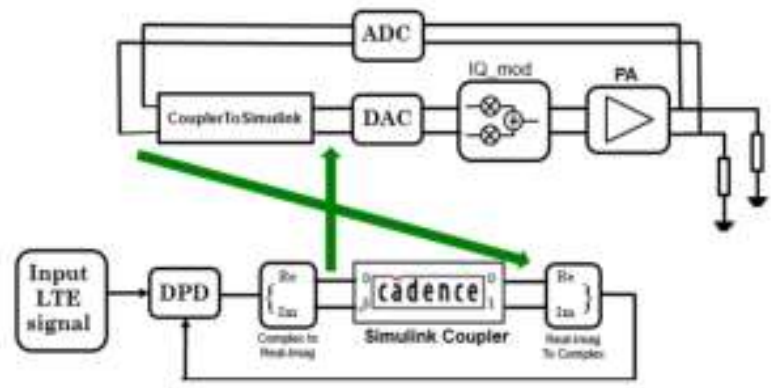

Fig. 5. Matlab/Cadence Platform prototype
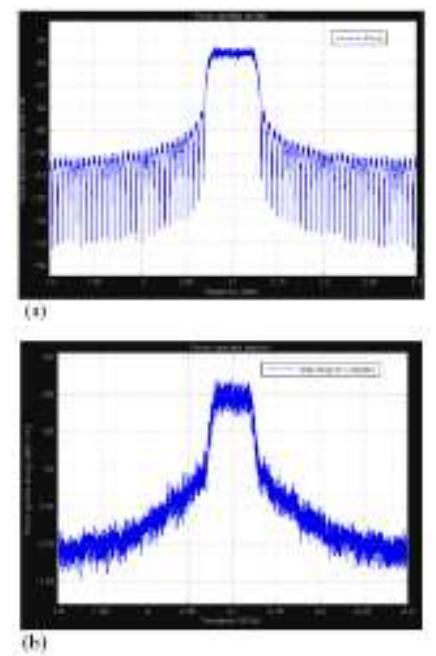

Fig. 6. Example simulation result of Matlab/Cadence Platform: (a) Input LTE Spectrum, (b) PA output Spectrum

\section{CONCLUSION}

This paper demonstrated workflow for implementation of three mixed signal platforms developed for the study of the 
accuracy/complexity trade-off of Digital Predistortion (DPD) algorithms and optimization of the power consumption for LTE base station applications. The project goal acheived was providing libraries in order to compare various PA models with various DPDs algorithms and be able to estimate with precision the performances and the power consumption of the whole amplification system. We used Co-simulation: MATLAB was used to model digital part, and Cadence used to model and design the RF analog part of the amplification system.

\section{REFERENCES}

[1] D. E. Root, J. Verspecht, D. Sharrit , J. Wood and A. Cognata, "Broadband poly-harmonic distorsion (PHD) behavioral models from fast automated simulations and large-signal vectorial network measurements", IEEE Trans. Microwave Theory Tech., vol. 53, no. 11, pp. 3656-3664, 2005.

[2] A. S. Tehrani, H. Cao, S. Afsardoost, T. Eriksson, M. Isaksson and C. Fager, "A comparative analysis of the complexity/accuracy tradeoff in power amplifier models", IEEE Trans. Microwav. Theory Tech., vol. 58, no.7, pp. 1510-1520, 2010.

[3] Ericsson Mobility Report, "On the pulse of the networked society", November

[4] B. Bangerter ; S. Talwar ; R. Arefi ; K. Stewart," Networks and devices for the 5G era", IEEE Communications Magazine, Volume:52, Issue: 2 , February 2014, Page(s): $90-96$.
[5] Ali Soltani Tehrani, Haiying Cao, Sepideh Afsardoost, Thomas Eriksson, Magnus Isaksson and Christian Fager, "A Comparative Analysis of the Complexity/Accuracy Tradeoff in Power Amplifier Behavioral Models", IEEE transactions on microwave theory and techniques, vol. 58, no. 6, june 2010.

[6] Mazen Abi Hussein, Olivier Venard, Bruno Feuvrie and Yide Wang, "Digital predistortion for RF power amplifiers: State of the art and advanced approaches", 11th International IEEE New Circuits and Systems Conference (NEWCAS), Paris, 16-19 June 2013.

[7] Hsing-Hung Chen, Ching-Shyang Maa, Yeong-Cheng Wang, and JiunnTsair Chen, "Joint polynomial and look-up-table power amplifierlinearization scheme," in 57th IEEE Semiannual Vehicular Tech. Conf. (VTC 2003), Jeju, Korea, April, 2003, vol. 2, pp. 13451349.

[8] S. Cripps, RF Power Amplifiers for Wireless Communications, Boston, MA: Artech House Inc., Academic Publishers, 1999.

[9] M. Hella and M. Ismail, RF CMOS Power Amplifiers: Theory, Design, Implementation. Norwell, MA: Kluwer Academic Publishers, 2002

[10] S. Bruss, "Linearization Methods," Class Lecture, Dep. Elect. Comput. Eng., University of California, Davis, April 2003

[11] Morgan, Ma, Kim, Zierdt, and Pastalan. "A Generalized Memory Polynomial Model for Digital Predistortion of RF Power Amplifiers". IEEE Trans. on Signal Processing, Vol. 54, No. 10, Oct. 2006

[12] Saleh, A.A.M., "Frequency-independent and frequency-dependent nonlinear models of TWT amplifiers," IEEE Trans. Communications, vol. COM-29, pp.1715-1720, November 1981.

[13] Cadence Design system, "Co-simulation with SpectreRF MATLAB/Simulink Tutorial”, Product Version 6.1.1, November 2006. 